\title{
BMJ Open A realist evaluation of patients' decisions to deprescribe in the EMPOWER trial
}

\author{
Philippe Martin, ${ }^{1,2}$ Cara Tannenbaum ${ }^{2,3}$
}

To cite: Martin $P$,

Tannenbaum C. A realist evaluation of patients' decisions to deprescribe in the EMPOWER trial. BMJ Open 2017;7:e015959. doi:10.1136/ bmjopen-2017-015959

- Prepublication history and additional material for this paper are available online. To view these files please visit the journal online (http://dx.doi. org/10.1136/bmjopen-2017015959).

Received 12 January 2017 Revised 21 February 2017 Accepted 8 March 2017

\section{CrossMark}

${ }^{1}$ Faculty of Pharmacy, Université de Montréal, Montreal, Québec, Canada

${ }^{2}$ Institut Universitaire de Gériatrie de Montréal, Montreal, Québec, Canada

${ }^{3}$ Université de Montréal,

Faculties of Medicine and Pharmacy, Montreal, Québec, Canada

Correspondence to Philippe Martin; philippe.martin@umontreal.ca

\section{ABSTRACT}

Background and objectives Successful mechanisms for engaging patients in the deprescribing process remain unknown but may include: (1) triggering motivation to deprescribe by increasing patients' knowledge and concern about medications; (2) building capacity to taper by augmenting self-efficacy and (3) creating opportunities to discuss and receive support for deprescribing from a healthcare provider. We tested these mechanisms during theEliminating Medications through Patient Ownership of End Results (EMPOWER) () trial and investigated the contexts that led to positive and negative deprescribing outcomes.

Design A realist evaluation using a sequential mixed methods approach, conducted alongside the EMPOWER randomised clinical trial.

Setting Community, Quebec, Canada.

Participants 261 older chronic benzodiazepine consumers, who received the EMPOWER intervention and had complete 6-month follow-up data.

Intervention Mailed deprescribing brochure on benzodiazepines.

Measurements Motivation (intent to discuss deprescribing; change in knowledge test score; change in beliefs about the risk-benefits of benzodiazepines, measured with the Beliefs about Medicines Questionnaire), capacity (self-efficacy for tapering) and opportunity (support from a physician or pharmacist).

Results The intervention triggered the motivation to deprescribe among $167(\mathrm{n}=64 \%)$ participants (mean age 74.6 years $\pm 6.3,72 \%$ women), demonstrated by improved knowledge (risk difference, 58.50\% (95\% Cl $46.98 \%$ to $67.44 \%)$ ) and increased concern about taking benzodiazepines (risk difference, $67.67 \%$ (95\% Cl $57.36 \%$ to $74.91 \%)$ ). Those who attempted to taper exhibited increased self-efficacy (risk difference, $56.90 \%$ (95\% Cl $45.41 \%$ to $65.77 \%)$ ). Contexts where the deprescribing mechanisms failed included lack of support from a healthcare provider, a focus on shortterm quality of life, intolerance to withdrawal symptoms and perceived poor health.

Conclusion Deprescribing mechanisms that target patient motivation and capacity to deprescribe yield successful outcomes in contexts where healthcare providers are supportive, and patients do not have internal competing desires to remain on drug therapy.

Trial registration number ClinicalTrials.gov: NCT01148186.
Strengths and limitations of this study

- Use of a mixed methods approach enabled us to explore the breadth, depth and complexity of the patient's experience of deprescribing.

- Use of the realist evaluation allowed us to investigate how the mechanisms underlying deprescribing interventions interact with specific contexts to yield positive or negative outcomes.

- This study was conducted alongside a large cluster randomised clinical trial.

\section{INTRODUCTION}

Deprescribing refers to the collaborative process of tapering, discontinuing, stopping or withdrawing medications in order to reduce adverse drug events and improve outcomes. $^{1-5}$ Deprescribing has many steps, ${ }^{13} 6$ with one key component being the engagement of patients in shared decision-making. ${ }^{1-15}$ Research suggests that older adults have conflicted feelings about medications ${ }^{414}$ : $78 \%$ of older adults believe that medications are necessary to improve health, but at the same time, $68 \%$ would like to reduce their current medication use, with $92 \%$ willing to stop a regular medication if advised to do so by their physician. ${ }^{14} \mathrm{~A}$ better understanding of the mechanisms that trigger patient motivation and capacity to engage in the deprescribing process could reduce the use of potentially inappropriate medications.

The aim of realist evaluation is to reveal how an intervention might generate different outcomes in different circumstances, and how mechanisms work in particular contexts, by enabling or motivating participants to make different choices. ${ }^{16}$ Educational strategies to increase patients' knowledge, beliefs and motivation are hypothesised to influence deliberate action on the part of the patient to curtail the use of a drug. ${ }^{10}$ However, what works, for whom, under which circumstances and why are questions that have never been explored systematically from the patient's 
point of view. Recent reviews on deprescribing call for a realist evaluation of large deprescribing trials to investigate how the mechanisms underlying deprescribing interventions interact with specific contexts to yield positive or negative outcomes. ${ }^{1718}$ The Eliminating Medications through Patient Ownership of End Results (EMPOWER) trial, which demonstrated a number-needed-to-treat of 4 for the effectiveness of mailing a benzodiazepine deprescribing brochure on complete cessation of benzodiazepines at 6 months, provides a timely opportunity to examine which deprescribing mechanisms worked under which circumstances. ${ }^{12}$

The initial theory underpinning the development of the EMPOWER intervention was that most, if not all, older adults are unaware of the age-related harms of taking benzodiazepine anti-anxiety drugs and sleeping pills. Side effects of sedative-hypnotics are well documented in the literature but rarely talked about in practice as being a potential cause of memory impairment, falls and fractures ${ }^{19-24}$ feared by many older adults. ${ }^{25}{ }^{26}$ Not understanding why medications should be discontinued is a patient barrier to deprescribing. ${ }^{47}$ As most patients are uninformed of the potential risks associated with the use of benzodiazepines, we hypothesised a linear behaviour change process whereby providing patients with an interactive educational brochure detailing associated risks, safer alternatives and steps for tapering would trigger patients' motivation, capacity and opportunity to initiate the deprescribing process through discussion of medication discontinuation with a healthcare provider.

This paper reports a realist evaluation of the deprescribing process from the patient's perspective. The realist evaluation tests the following mechanisms: (1) whether the EMPOWER intervention triggered patients' motivation to deprescribe by increasing knowledge and concern about benzodiazepines; (2) augmented patients' capacity and self-efficacy to taper benzodiazepines and (3) created opportunities for the patient to discuss and receive support from a healthcare provider to engage in the deprescribing process. We also determined in which contexts successful and failed deprescribing outcomes occurred.

\section{METHODS}

\section{Study design}

A realist evaluation was conducted alongside the EMPOWER randomised controlled trial. ${ }^{12}$ This report follows online supplementary material 2 RAMESES II guidelines for realist evaluation. ${ }^{16}$ The approach was chosen to inform the implementation of future deprescribing initiatives by examining the possible causes and contextual factors associated with change. ${ }^{28}$ Realist evaluation is a theory-based, sequential mixed methods approach that seeks to gain a deeper understanding of contexts, mechanisms and outcomes. This is accomplished through the identification and examination of underlying generative mechanisms (M) associated with the intervention or programme, the conditions or contexts $(\mathrm{C})$ under which the mechanisms operate, and the pattern of outcomes $(\mathrm{O})$ produced. These may be expressed as linked Contexts-Mechanisms-Outcomes configurations (or $\mathrm{C}+\mathrm{M}=\mathrm{O}$ ) ${ }^{28}$ In this case, the $(\mathrm{C})$ consist of all internal and external factors that can influence the deprescribing process and the $(\mathrm{O})$ refer to whether or not the deprescribing intervention was successful. The (M) that we aimed to test were whether the EMPOWER brochure: (1) triggered older adults' motivation to deprescribe by increasing knowledge and concern about benzodiazepines; (2) built capacity to taper by augmenting self-efficacy and (3) drove opportunities to receive support from a healthcare provider to deprescribe.

The study was approved by the Institut Universitaire de Gériatrie de Montréal Ethics Committee in Montreal, Quebec, Canada.

\section{Environment surrounding the evaluation}

The EMPOWER trial was a pragmatic randomised trial that examined the effectiveness of a direct-to-consumer, written educational brochure mailed directly to patients on subsequent discontinuation of sedative-hypnotic medication. ${ }^{29}$ The EMPOWER trial was rolled out between July 2010 and November 2013, with community-dwelling participants randomly recruited via pharmacists located within a $200 \mathrm{~km}$ radius of the Montreal urban area in Quebec, Canada. Participants were 303 older, community-dwelling, chronic users of benzodiazepine medication and agreed to home visits and telephone follow-up interviews by the research team. All benzodiazepine prescriptions for seniors were covered under the publicly financed drug plan in the province of Quebec, excluding the programme's deductible (if applicable). Provincial governments covered physician reimbursements for patient visits, and drug dispensing fees for pharmacists, as part of Canada's universal healthcare programme.

\section{The EMPOWER intervention}

The eight-page EMPOWER brochure, available at http://www.criugm.qc.ca/fichier/pdf/BENZOeng.pdf, ${ }^{30}$ aims to promote active learning by incorporating and using constructivist learning principles. ${ }^{31}$ The brochure includes a self-assessment component and presentation of the evidence-based risks associated with benzodiazepine use in an effort to elicit cognitive dissonance. ${ }^{10}$ Elements of social comparison theory, ${ }^{32}$ through the use of peer champion stories, are also integrated in the intervention. The brochure provides a self-guided tapering schedule, consisting of a visual tapering protocol showing pictures of full pills, halved pills and quartered pills. ${ }^{30}$

\section{Evaluation of mechanisms and contexts}

The mechanisms embedded in the EMPOWER intervention are based on Michie $e t a l$ s behaviour change wheel, ${ }^{33}$ targeting motivation, capacity and opportunity. Michie $e t$ al define motivation as the mental process that energises 
Table 1 Programme mechanisms embedded in EMPOWER intervention

\begin{tabular}{|c|c|c|c|}
\hline \multirow{2}{*}{$\begin{array}{l}\text { Mechanisms } \\
\text { Increase motivation } \\
\text { to deprescribe by } \\
\text { changing knowledge } \\
\text { and beliefs }\end{array}$} & \multicolumn{3}{|c|}{ Components of the EMPOWER brochure } \\
\hline & $\begin{array}{l}\text { Messaging on the front page } \\
\text { 'You May be at Risk' to raise } \\
\text { awareness of the harms of } \\
\text { benzodiazepines }\end{array}$ & $\begin{array}{l}\text { Interactive knowledge test } \\
\text { with four true/false questions } \\
\text { and answers about the harms } \\
\text { of benzodiazepines, aimed at } \\
\text { increasing knowledge }\end{array}$ & $\begin{array}{l}\text { Information about changes in drug } \\
\text { metabolism with age that can lead } \\
\text { to a higher risk of side effects, } \\
\text { meant to change beliefs and elicit } \\
\text { concern about the safety of the } \\
\text { medication in older adults }\end{array}$ \\
\hline $\begin{array}{l}\text { Drive opportunities to } \\
\text { discuss and initiate } \\
\text { deprescribing with a } \\
\text { healthcare provider }\end{array}$ & $\begin{array}{l}\text { Instruction to 'Please consult } \\
\text { your doctor or pharmacist before } \\
\text { stopping any medication' in a } \\
\text { large red box }\end{array}$ & $\begin{array}{l}\text { Logos on the brochure } \\
\text { provide source credibility } \\
\text { for the patient to initiate } \\
\text { conversations }\end{array}$ & $\begin{array}{l}\text { The printed format of the eight- } \\
\text { page brochure makes it an effective } \\
\text { knowledge transfer piece to take } \\
\text { and show to a healthcare provider }\end{array}$ \\
\hline
\end{tabular}

and directs behaviours. Capability refers to the psychological and physical capacity of the individual to engage in the behaviour. Opportunity refers to the internal and external factors that permit or promote a behaviour to happen, and include both the physical and social environment of the individual. Table 1 links the programme mechanisms to the corresponding intervention components.

The evaluation of mechanisms and contexts consisted of quantitative data collection and analysis, qualitative data collection and analysis and triangulation of the quantitative and qualitative results. ${ }^{34}$ Data collection was conducted between July 2010 and November 2013 as part of the EMPOWER clinical trial. Analysis, triangulation and refinement of the Context-Mechanism-Outcome configuration took place subsequent to completion of the trial.

\section{Data collection methods}

Quantitative data included preintervention and 1-week postintervention information on knowledge about benzodiazepine-related harms, beliefs about the necessity of taking benzodiazepines versus concern about harms, self-efficacy for tapering and intent to discuss deprescribing with a healthcare provider. We measured gains in knowledge with the four true or false questions listed in the 'Test Your Knowledge' section of the questionnaire. ${ }^{2930}$ Correct answers were summed to a maximum of 4 points, and answers were compared prior to and after receiving the intervention. Participants' beliefs about consuming benzodiazepines were measured with the Beliefs about Medicines Questionnaire (BMQ-Specific) at both time points. The BMQ-Specific consists of two validated five-item subscales assessing the respondents' perceptions about the necessity and concerns associated with taking benzodiazepines. ${ }^{35}$ Participants indicate their degree of agreement with each statement on a five-point Likert scale ( $1=$ strongly disagree, $5=$ strongly agree). Scores are summed into their respective subcategory
(5-25 point scale) with higher scores indicating stronger beliefs. Risk perception was assessed using a single question 1-week postintervention in which participants were asked whether they perceived the same, increased or no risk from consumption of their benzodiazepine following the intervention. In order to determine whether the EMPOWER brochure increased capacity to taper by augmenting self-efficacy, we measured self-efficacy for tapering on the Medication Reduction Self-efficacy scale, which allows the respondent to rate on a scale of 0 to 100 their degree of confidence for tapering benzodiazepines. ${ }^{36}$ Higher scores indicate greater self-efficacy. Participants were also asked to indicate (yes/no) postintervention if they had spoken to or intended to discuss medication discontinuation with their doctor and/or pharmacist. Health status was assessed at baseline using the first item of the Short-Form-12 Health Survey and dichotomised by categorising poor to fair responses as poor health. $^{37}$

Qualitative data were collected after the 6-month follow-up, using semistructured interviews conducted at participants' homes to determine the contexts under which the deprescribing mechanisms succeeded or failed. Twenty-one participants were strategically sampled for the interviews using a contrast sample design, based on cessation of benzodiazepines (yes or no) combined with intent to discuss tapering (yes or no). ${ }^{38}$ Interviews lasted approximately 1 hour, were recorded with consent and professionally transcribed verbatim. The interviews were based on a pre-established discussion guide, the major themes of which included initial reactions to the intervention, reasons underlying the decision to taper, experience with the tapering process and personal interactions with healthcare providers (see online supplementary material 1).

\section{Analysis}

The three mechanisms of increasing motivation, capacity and opportunity were tested using quantitative analysis. 
Participants with complete follow-up data were included in the quantitative analysis $(\mathrm{n}=261$, mean age $74.6 \pm 6.3$, $72 \%$ women). Data were described and compared using means with SD and independent t-tests for continuous data, and percentages and $\chi^{2}$ tests for categorical data, according to each of three outcomes: intent to deprescribe with successful discontinuation, intent to deprescribe with failed discontinuation and no intent to deprescribe. Individuals who achieved a dose reduction were classified as intent to deprescribe with failed discontinuation. Participant changes in knowledge, in the BMQ necessity and concerns subscales and in self-efficacy scores for tapering were computed from baseline to postintervention. Risk differences with 95\% CIs were calculated for the proportion of participants in each group who demonstrated increased knowledge, heightened concern about benzodiazepine use and augmented self-efficacy for tapering. The statistical significance for all analyses was set at $\mathrm{p}<0.05$ (two-sided) ${ }^{39}$ SPSS V.21.0 was used for all analyses.

Qualitative data from the semistructured interviews were analysed using thematic content analysis to explore the contexts under which the programme mechanisms led to positive or negative outcomes. ${ }^{40}$ Discourses were contrasted according to whether participants discontinued benzodiazepines and/or expressed the intent to discuss discontinuation. Interviews were coded using Dedoose software. Contextual themes were derived from the data and supported by quotes. Initially, two researchers independently read the transcripts and field notes, then collaboratively developed first order codes, which were subsequently verified by double coding. Second order thematic coding was performed for the purpose of building concepts.

Quantitative and qualitative results about context were combined and analysed in an iterative fashion through use of a triangulation protocol using a convergence coding matrix, ${ }^{41}$ as described by Farmer et al. ${ }^{42}$ The convergence matrix served to inform which contexts favourably or unfavourably influenced a patient's decision to deprescribe based on agreement, partial agreement or dissonance between the quantitative and qualitative data. ${ }^{41} 42$ Differences were adjudicated via discussion and consensus. ${ }^{42}$ The convergence-coding matrix is available from the authors on request.

\section{RESULTS}

\section{Linking mechanisms to outcomes}

The mechanism of triggering motivation to deprescribe occurred in 167 of 261 individuals (64\%) who received the EMPOWER intervention (table 2).Participants who expressed an intent to deprescribe postintervention had improved knowledge (risk difference, $58.50 \%$ (95\% CI $46.98 \%$ to $67.44 \%)$ ), lower perceived necessity scores (risk difference, $56.03 \%$ (95\% CI $44.63 \%$ to $64.81 \%$ )), increased concern (risk difference, $67.67 \%$ (95\% CI $57.36 \%$ to $74.91 \%$ )) and a greater perception of risk about their benzodiazepine medication than those who were not motivated to attempt deprescribing (risk difference, $35.14 \%$ (95\% CI $23.06 \%$ to $45.39 \%$ )). Individuals who decided to deprescribe exhibited higher capacity for tapering after receipt of the EMPOWER brochure, with enhanced self-efficacy compared with those in whom the intervention did not trigger motivation (risk difference, $56.90 \%$ (95\% CI $45.41 \%$ to $65.77 \%$ )) (table 2). Approximately half of individuals with augmented motivation and capacity to deprescribe initiated a conversation with their physician, and $25 \%$ spoke to a pharmacist about deprescribing. Neither postintervention self-efficacy scores nor creating the opportunity to discuss deprescribing with a healthcare provider distinguished between positive or negative outcomes among motivated individuals.

\section{Contexts associated with positive deprescribing outcomes}

Table 3 shows the results of the qualitative analysis, describing the contexts that enabled the EMPOWER mechanisms to achieve positive deprescribing outcomes. Favourable personal contexts included stable health status and a positive outlook on ageing. Individuals who were not dealing with acute health issues were more receptive to tapering off benzodiazepines, as were individuals who prioritised long life expectancy over the short-term benefits of continued use or the transient discomfort associated with deprescribing benzodiazepines. Individuals who succeeded in tapering had the highest baseline self-efficacy for being able to discontinue (table 2). External influences associated with successful discontinuation were previous and ongoing support or encouragement from a healthcare provider (table 3).

\section{Contexts in which the EMPOWER mechanisms failed}

Thirty-six per cent of the participants in the trial reported no desire to deprescribe after receipt of the EMPOWER brochure. These individuals showed no gain in knowledge and no increase in perceived risk post-intervention (table 2). Failure for the EMPOWER intervention to elicit motivation to deprescribe was more likely among individuals who reported poor health $(40 \%$ vs $28 \%$, $12.28 \%$ ( $95 \%$ CI $0.44 \%$ to $24.18 \%$ )). During the qualitative interviews, participants dealing with ongoing health issues expressed a strong reliance on benzodiazepines for everyday coping (table 4). Other contexts associated with the decision not to attempt deprescribing included previous reassurance by a physician that benzodiazepines were safe or necessary and the belief that the benefits of benzodiazepines outweighed the risks for immediate symptom relief (table 4). Contexts that led participants to abort the deprescribing process once they showed initial motivation, capacity and opportunity to deprescribe included the lack of support from a healthcare provider, intolerance to withdrawal symptoms and a sudden loss of confidence to live without sleeping pills (table 4 ). 


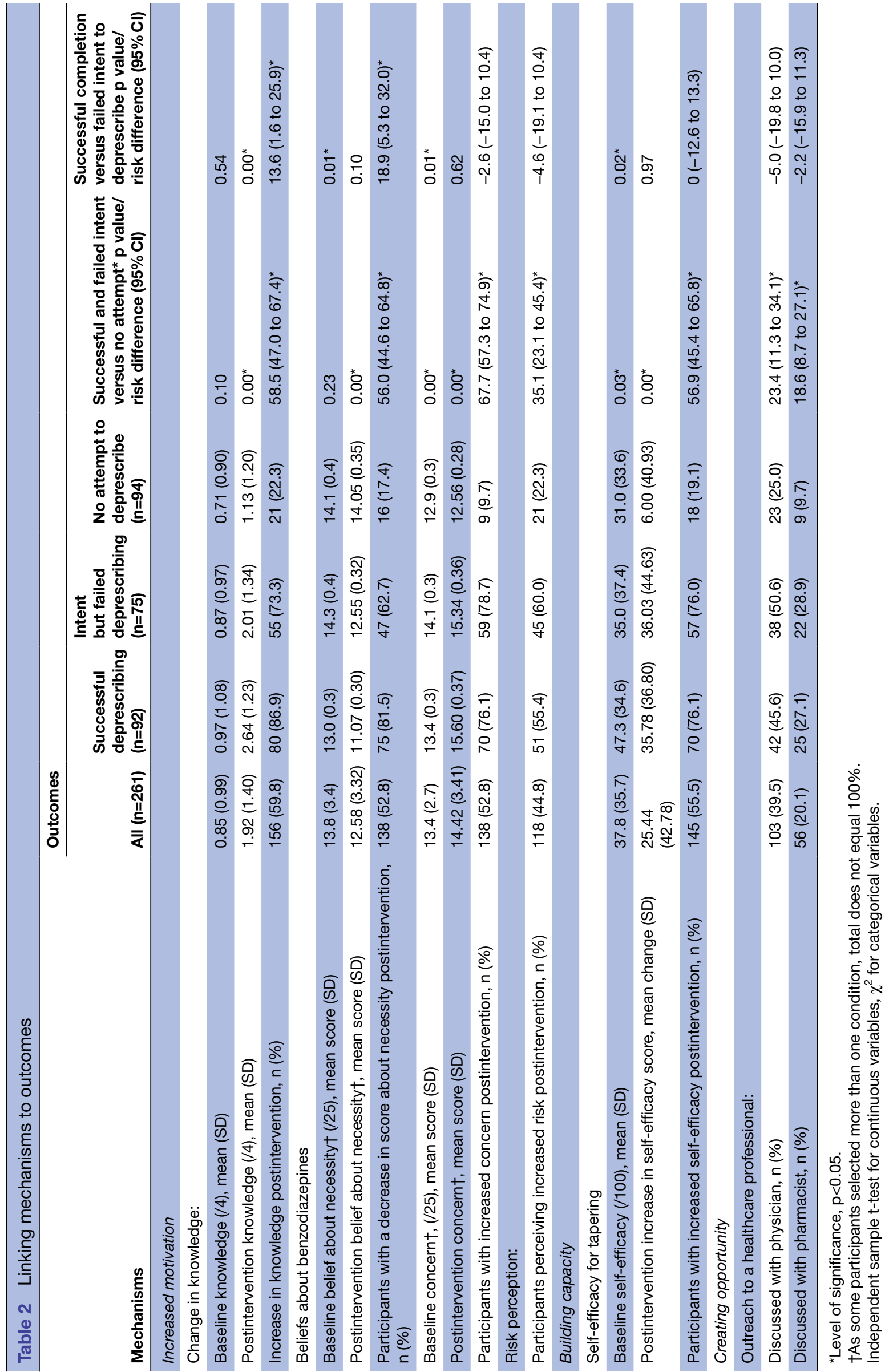


Table 3 Contexts associated with positive outcomes

\begin{tabular}{|c|c|c|c|c|}
\hline \multirow[b]{2}{*}{ Contexts } & \multicolumn{4}{|l|}{ Outcomes } \\
\hline & $\begin{array}{l}\text { Successful } \\
\text { deprescribing } \\
(\mathrm{n}=7)\end{array}$ & $\begin{array}{l}\text { Failed } \\
\text { deprescribing } \\
(\mathrm{n}=7)\end{array}$ & $\begin{array}{l}\text { No attempt to } \\
\text { deprescribe } \\
(\mathrm{n}=7)\end{array}$ & Supporting citation \\
\hline $\begin{array}{l}\text { Previous support from } \\
\text { physician/positive } \\
\text { attitude towards } \\
\text { discontinuation }\end{array}$ & $5(71 \%)$ & $4(57 \%)$ & $1(14 \%)$ & $\begin{array}{l}\text { 'He (my doctor) told me the drug was not good } \\
\text { for me and that I could experience side effects } \\
\text { while taking it'. (72-year-old man, successful } \\
\text { taper) }\end{array}$ \\
\hline Stable health status & $5(71 \%)$ & $4(57 \%)$ & $2(29 \%)$ & $\begin{array}{l}\text { 'I don't have as much pain as I used to. It's } \\
\text { now under control so it was easier for me to } \\
\text { stop. Before-no way'. (68-year-old woman, } \\
\text { successful taper) }\end{array}$ \\
\hline $\begin{array}{l}\text { Certainty and confidence } \\
\text { about tapering } \\
\text { (postintervention) }\end{array}$ & $6(86 \%)$ & $4(57 \%)$ & $1(14 \%)$ & $\begin{array}{l}\text { 'I persuaded myself that I needed to get rid } \\
\text { of this, no matter what'. (84-year-old man, } \\
\text { successful taper) }\end{array}$ \\
\hline $\begin{array}{l}\text { Perception of increased } \\
\text { risk }\end{array}$ & $6(86 \%)$ & $5(71 \%)$ & $1(14 \%)$ & $\begin{array}{l}\text { 'My physician told me it (the drugs) could cost } \\
\text { me my memory. My memory has become very } \\
\text { important to me'. ( } 79 \text {-year-old man, successul } \\
\text { taper) }\end{array}$ \\
\hline $\begin{array}{l}\text { Lack of psychological } \\
\text { attachment }\end{array}$ & $5(71 \%)$ & $3(43 \%)$ & $1(14 \%)$ & $\begin{array}{l}\text { 'I understood I could stop taking it (after I read } \\
\text { the brochure), that it was not an obligation (to } \\
\text { take it)'. (72-year-old woman, successful taper) }\end{array}$ \\
\hline $\begin{array}{l}\text { Positive outlook on } \\
\text { ageing }\end{array}$ & $3(43 \%)$ & $1(14 \%)$ & 0 & $\begin{array}{l}\text { 'At my age I don't believe in miracles such as } \\
\text { being able to sleep for } 8,9 \text { or } 10 \text { hours each } \\
\text { night. It would be impossible for me, so I } \\
\text { content myself with the hours of sleep I get'. } \\
\text { (84-year-old man, successful taper) }\end{array}$ \\
\hline $\begin{array}{l}\text { Tapering tool provides } \\
\text { support }\end{array}$ & $5(71 \%)$ & $3(43 \%)$ & 0 & $\begin{array}{l}\text { 'In the past I tried to stop the pill all at once. } \\
\text { But using the tapering tool, I understood } \\
\text { that it need to be a gradual and not a drastic } \\
\text { process'. (84-year-old man, successful taper) }\end{array}$ \\
\hline $\begin{array}{l}\text { Supportive healthcare } \\
\text { provider }\end{array}$ & $3(43 \%)$ & $2(29 \%)$ & 0 & $\begin{array}{l}\text { 'When I told my doctor I wanted to stop, he } \\
\text { said, 'no problem, let's do it'. (87-year-old } \\
\text { woman, successful taper) }\end{array}$ \\
\hline
\end{tabular}

Refining the context-mechanism-outcome configuration for deprescribing interventions

The initial context-mechanism-outcome configuration that drove the development of the EMPOWER intervention was a simple, linear progression along different stages of readiness to deprescribe, similar to Prochaska \& DiClemente's transtheoretical model of change (figure 1A). ${ }^{43}$ We believed that the EMPOWER brochure would trigger motivation and capacity to deprescribe, moving patients from precontemplation about deprescribing to action and maintenance, by increasing knowledge about the harms of benzodiazepines, enhancing self-efficacy and creating opportunities to discuss deprescribing with a healthcare professional. We assumed that the healthcare provider would provide a supportive context, encouraging the patient to deprescribe, thereby yielding a positive outcome. This initial configuration oversimplified the stages through which individuals transitioned after receiving the deprescribing intervention. Figure 1B depicts a revised, non-linear context-mechanismoutcome configuration that takes into account the complexity of internal and external contexts on initiating and completing the deprescribing process from the consumer's perspective. The revised model recognises that new information influences beliefs and actions only if the information generates a desire strong enough not to be overwhelmed by competing motivations arising from other sources. In many instances, the desire for risk reduction, which was the prime motivator behind the development of the EMPOWER intervention, did not supersede concerns about symptom recurrence, or other psychological and health factors, as well as interpersonal relationships with healthcare providers, which played critical contextual roles in the outcome of the intervention.

\section{DISCUSSION}

This realist evaluation tested the mechanisms embedded in the EMPOWER intervention and showed that motivation and capacity to deprescribe were triggered in $64 \%$ of older chronic benzodiazepines consumers, the majority of whom created an opportunity to discuss 
Table 4 Contexts associated with negative outcomes

\begin{tabular}{|c|c|c|c|c|}
\hline Key theme & $\begin{array}{l}\text { Successful } \\
\text { deprescribing } \\
(n=7)\end{array}$ & $\begin{array}{l}\text { Failed } \\
\text { deprescribing } \\
(n=7)\end{array}$ & $\begin{array}{l}\text { No attempt to } \\
\text { deprescribe } \\
(n=7)\end{array}$ & Supporting citation \\
\hline $\begin{array}{l}\text { Previous discouragement } \\
\text { from physician }\end{array}$ & $1(14 \%)$ & $1(14 \%)$ & $5(71 \%)$ & $\begin{array}{l}\text { 'I asked him (my doctor), 'Are there any of } \\
\text { my medications I could stop?' He told me, } \\
\text { 'No, we're not taking anything away, you are } \\
\text { doing well'. I then told him my medication was } \\
\text { getting very expensive to which he replied, } \\
\text { 'You know Mr., life is priceless'. (75-year-old } \\
\text { man, no intent to taper) }\end{array}$ \\
\hline Poor health status & 0 & $1(14 \%)$ & $4(57 \%)$ & $\begin{array}{l}\text { 'If anyone stops my pills, poof, I would die for } \\
\text { sure because of my poor health'. (70-year-old } \\
\text { woman, no intent to taper) }\end{array}$ \\
\hline $\begin{array}{l}\text { Unquestioning belief in } \\
\text { their physician }\end{array}$ & $1(14 \%)$ & $1(14 \%)$ & $3(43 \%)$ & $\begin{array}{l}\text { 'If you take all your pills as prescribed, you'll } \\
\text { never have problems in your life [...] When my } \\
\text { doctor prescribes something for me, I know } \\
\text { it's not junk, I know it's good for me. And I } \\
\text { don't question it'. (72-year-old man, no intent } \\
\text { to taper) }\end{array}$ \\
\hline $\begin{array}{l}\text { Lack of perception of } \\
\text { personal risk }\end{array}$ & $1(14 \%)$ & 2 (29\%) & $5(71 \%)$ & $\begin{array}{l}\text { 'I recall that he (my doctor) told me that in the } \\
\text { long-term my benzodiazepine could affect } \\
\text { my memory. But my memory is fantastic'. } \\
\text { (72-year-old man, no intent to taper) }\end{array}$ \\
\hline $\begin{array}{l}\text { Reliance on medication for } \\
\text { coping/everyday function }\end{array}$ & $1(14 \%)$ & $1(14 \%)$ & $4(57 \%)$ & $\begin{array}{l}\text { 'Without this medication, I know that my life } \\
\text { would be plagued by anxiety, of this I am } \\
\text { certain'. (68-year-old woman, no intent to } \\
\text { taper) }\end{array}$ \\
\hline $\begin{array}{l}\text { Quality of life focus during } \\
\text { end of life }\end{array}$ & 0 & 2 (29\%) & $3(43 \%)$ & $\begin{array}{l}\text { 'At my age I don't care about the risks. I } \\
\text { don't care if I live to } 100 \text { or not'. (85-year-old } \\
\text { woman, failed tapering) }\end{array}$ \\
\hline $\begin{array}{l}\text { Intolerance to recurrence } \\
\text { of symptoms/withdrawal } \\
\text { effects }\end{array}$ & 0 & $5(71 \%)$ & - & $\begin{array}{l}\text { 'When I decreased the dose I started getting } \\
\text { headaches. I felt miserable not being able } \\
\text { to sleep at night'. (85-year-old man, failed } \\
\text { tapering) }\end{array}$ \\
\hline $\begin{array}{l}\text { Loss of confidence to } \\
\text { complete the tapering } \\
\text { process (postintervention) }\end{array}$ & 0 & $4(57 \%)$ & $4(57 \%)$ & $\begin{array}{l}\text { 'I knew that l'd be in trouble without my pills. } \\
\text { It's been a long time now. How can I put it in } \\
\text { words? If I ran out of pills l'd be in trouble'. } \\
\text { (85-year-old man, failed tapering) }\end{array}$ \\
\hline
\end{tabular}

deprescribing with a healthcare provider. These findings support the theory that provision of new knowledge about medication harms can raise concern and augment patients' self-efficacy to deprescribe. However, the analysis also indicates that human motivation to deprescribe is complex and unstable. A variety of internal and external contexts can interfere with the decision to deprescribe. Internal influences include perceptions about one's health status, long-term health goals, fear of symptom recurrence and psychological attachment to the drug. The main external influence that blocks consumer-directed deprescribing mechanisms is the lack of support from a healthcare provider.
Our findings contribute to the literature by illustrating that linear progression along different stages of readiness to deprescribe does not fully explain successful deprescribing from the patient's perspective. This conclusion is consistent with other critiques of the transtheoretical model, which claim that the stages of readiness are arbitrary, that human beings do not make logical and stable plans to change their behaviour and that setbacks can occur along the trajectory of change. ${ }^{44}$ Education appears to be necessary but insufficient for many individuals, and new strategies will be needed to trigger deprescribing in prohibitive contexts where the EMPOWER mechanisms failed. As capacity and motivations change over time, 


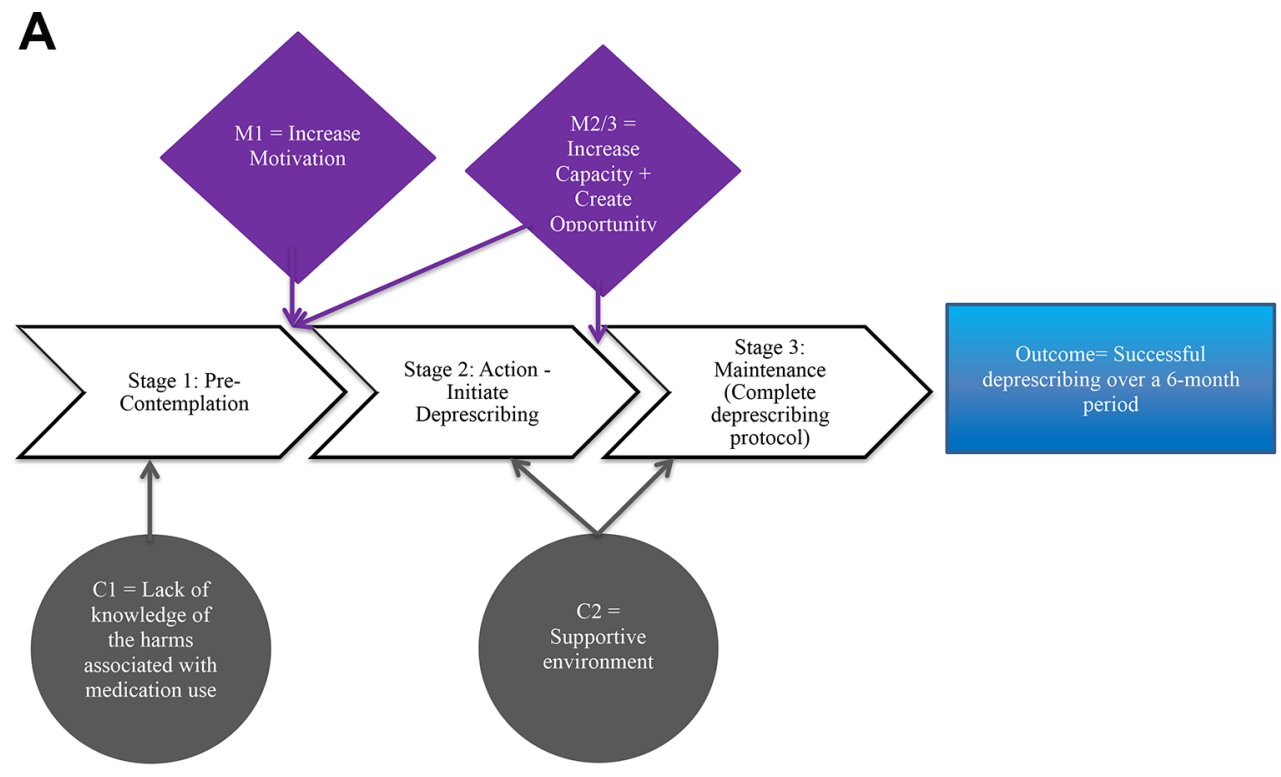

B

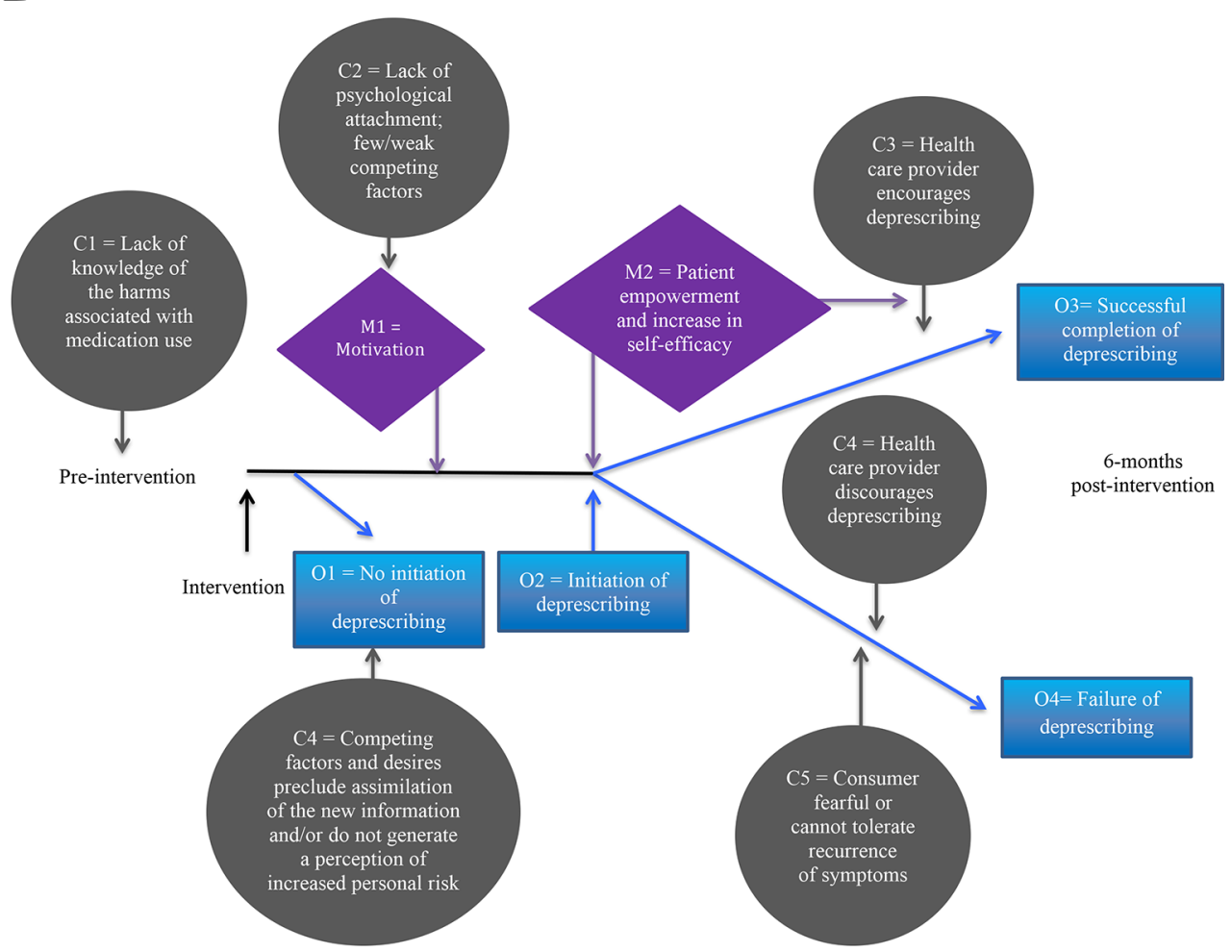

Figure 1 (A) Initial deprescribing context-mechanism-outcome configuration. (B) Refined deprescribing context-mechanismoutcome configuration. 
reminders and ongoing discussions about the risks of inappropriate medications may progressively trigger and sustain patients' commitments to engage in the deprescribing process. Some competing factors may wane, such as poor health. Offering cognitive behavioural therapy to patients during the most difficult last quarter period of the tapering protocol may augment self-efficacy for overcoming withdrawal symptoms. ${ }^{36}$ Interventions can be directed at healthcare providers who discourage deprescribing efforts. Continuing medical education to inform health providers about the mounting evidence on the harms of benzodiazepine use may curtail the phenomenon of physicians who continue to promote the use of inappropriate medication. ${ }^{20}{ }^{45}$ Future research directions should also include measurement of cognitive dissonance, which lies at the heart of constructivist learning. ${ }^{46}$ Methods to measure cognitive dissonance, defined as a feeling of tension between two sets of competing beliefs and motivations, may shed light on the way in which tensions about deprescribing are played out and drive behaviour change. ${ }^{4647}$ As we did not directly ask patients if they felt internal tension, we were unable to record feelings or processes of cognitive dissonance.

Use of a mixed methods approach enabled us to explore the breadth, depth and complexity of the patient's experience of deprescribing from a social, behavioural and health perspective, allowing stronger inferences about the various contexts affecting patients' decisions than could be achieved through a quantitative or qualitative lens alone. ${ }^{48}$ However, other mechanisms and contexts may trigger motivation to deprescribe beyond what is described in this realist evaluation. One untested mechanism is provision of information about the lack of drug benefits in certain populations, such as statins to reduce cholesterol levels in palliative care patients with limited life expectancy. ${ }^{49}$ Another challenge that we experienced during the conduct of this realist evaluation was differentiating between the mechanisms and contexts associated with deprescribing. ${ }^{51}$ For instance, when participants stated that their physician or pharmacist undermined their decision to deprescribe, it was clear this factor changed the reasoning of the participants. However, we were not sure whether this factor should be labelled as a mechanism or a context. Since the mechanism of action is defined as the 'how' behind the generation of outcomes, we initially thought that healthcare provider support was a mechanism that brought about deprescribing. ${ }^{51}$ On iterative reflection and discussion of the $\mathrm{C}-\mathrm{M}-\mathrm{O}$ configurations, we came to the conclusion that healthcare provider support was actually a context that enabled or hindered the consumer's motivation, capacity and opportunity to deprescribe, as triggered by the EMPOWER intervention. We drew this conclusion by subscribing to Pawson and Tilley's initial approach to realist evaluation, which seeks to identify mechanisms at the level of the individual's human reasoning. ${ }^{52}$ Others such as Dalkin et al posit that interpersonal relationships between stakeholders are a key factor that influence human reasoning, and argue that mechanisms can also be evaluated through the social lens of human and systems interactions. ${ }^{51}$ Deprescribing in particular is a complex social process that involves patients, prescribers and pharmacists, so our analysis may be faulted by some for studying the consumer's decision-making processes in isolation. For this reason, we chose not to make a table listing discrete $\mathrm{C}-\mathrm{M}-\mathrm{O}$ relationships in this paper but instead focused on broadly describing and testing the mechanisms embedded in the EMPOWER intervention and outlining the different personal, interpersonal and external contexts that led to positive or negative outcomes. We created figure 1A,B with difficulty, and some scepticism about whether these complex interactions could be illustrated in simple form. As the field of realist evaluation evolves, new terminology and formats may emerge that better capture a way of graphically illustrating the science of human interactions and behaviour change.

In conclusion, this realist evaluation conducted alongside a clinical trial provides important insights about deprescribing from the patient's perspective and increases current understanding about the specific mechanisms and contexts that generate positive or negative outcomes when attempting to engage patients in curbing the overuse and potentially inappropriate use of medicines.

Acknowledgements We wish to acknowledge the work of Anne-Sophie Michaud and Anastasia Soboleva who conducted the in-home interviews and helped in identifying first order coding for the qualitative portion of the manuscript. Additionally, we would like to thank Johanne Collin for her advice in devising the general strategy for the qualitative interviews. We express gratitude to all the participants and pharmacists who took part in this trial. Particular thanks are offered to the Pharmacy Services Department of the Jean Coutu Group (PJC) Inc. for their collaboration and support.

Contributors PM and CT contributed to the study concept and design, analysis and interpretation of data, drafting of the manuscript, critical revision of the manuscript for important intellectual content and final approval of the version to be published.

Funding This work was supported by Operating Grant 0TG-88591 from the Canadian Institutes of Health Research (CIHR). PM received a doctoral bursary from the FRQS. CT was supported by a Senior Scientist Career Award from the FRQS. The above funding organisations had no role in the design and conduct of the study; collection, management, analysis and interpretation of the data; preparation, review or approval of the manuscript; or the decision to submit the manuscript for publication.

Competing interests None declared.

Ethics approval Institut Universitaire de Gériatrie de Montréal Ethics Committee.

Provenance and peer review Not commissioned; externally peer reviewed.

Data sharing statement Dataset is available upon request to the corresponding author.

Open Access This is an Open Access article distributed in accordance with the Creative Commons Attribution Non Commercial (CC BY-NC 4.0) license, which permits others to distribute, remix, adapt, build upon this work non-commercially, and license their derivative works on different terms, provided the original work is properly cited and the use is non-commercial. See: http://creativecommons.org/licenses/by-nc/4.0/

(c) Article author(s) (or their employer(s) unless otherwise stated in the text of the article) 2017. All rights reserved. No commercial use is permitted unless otherwise expressly granted.

\section{REFERENCES}

1. Jansen J, Naganathan V, Carter SM, et al. Too much medicine in older people? deprescribing through shared decision making. BMJ 2016;353:i2893.

2. Scott IA, Hilmer SN, Reeve E, et al. Reducing inappropriate polypharmacy: the process of deprescribing. JAMA Intern Med 2015;175:827-34. 
3. Frank $\mathrm{C}$, Weir E. Deprescribing for older patients. CMAJ 2014;186:1369-76.

4. Reeve E, To J, Hendrix I, et al. Patient barriers to and enablers of deprescribing: a systematic review. Drugs Aging 2013;30:793-807.

5. Woodward MC. Deprescribing: achieving Better Health Outcomes for older people through reducing medications. J Pharm Pract Res 2003;33:323-8.

6. Scott IA, Gray LC, Martin JH, et al. Deciding when to stop: towards evidence-based deprescribing of drugs in older populations. Evid Based Med 2013;18:121-4.

7. Iyer S, Naganathan V, McLachlan AJ, et al. Medication withdrawal trials in people aged 65 years and older: a systematic review. Drugs Aging 2008;25:1021-31.

8. Van der Cammen TJ, Rajkumar C, Onder G, et al. Drug cessation in complex older adults: time for action. Age Ageing 2014;43:20-5.

9. Declercq T, Petrovic M, Azermai M, et al. Withdrawal versus continuation of chronic antipsychotic drugs for behavioural and psychological symptoms in older people with dementia. Cochrane Database Syst Rev 2013;3:CD007726.

10. Martin P, Tamblyn R, Ahmed S, et al. A drug education tool developed for older adults changes knowledge, beliefs and risk perceptions about inappropriate benzodiazepine prescriptions in the elderly. Patient Educ Couns 2013;92:81-7.

11. Ostini R, Hegney D, Jackson C, et al. Systematic review of interventions to improve prescribing. Ann Pharmacother 2009;43:502-13.

12. Tannenbaum C, Martin P, Tamblyn R, et al. Reduction of inappropriate benzodiazepine prescriptions among older adults through direct patient education: the EMPOWER cluster randomized trial. JAMA Intern Med 2014;174:890-8.

13. Cook JM, Biyanova T, Masci C, et al. Older patient perspectives on long-term anxiolytic benzodiazepine use and discontinuation: a qualitative study. J Gen Intern Med 2007;22:1094-100.

14. Reeve E, Wiese MD, Hendrix I, et al. People's attitudes, beliefs, and experiences regarding polypharmacy and willingness to Deprescribe. J Am Geriatr Soc 2013;61:1508-14.

15. Pollmann AS, Murphy AL, Bergman JC, et al. Deprescribing benzodiazepines and Z-drugs in community-dwelling adults: a scoping review. BMC Pharmacol Toxicol 2015;16:19.

16. Wong G, Westhorp G, Manzano A, et al. RAMESES II reporting standards for realist evaluations. BMC Med 2016;14:96.

17. Johansson T, Abuzahra ME, Keller S, et al. Impact of strategies to reduce polypharmacy on clinically relevant endpoints: a systematic review and meta-analysis. Br J Clin Pharmacol 2016;82:532-48.

18. Page AT, Clifford RM, Potter K, et al. The feasibility and effect of deprescribing in older adults on mortality and health: a systematic review and meta-analysis. Br J Clin Pharmacol 2016;82:583-623.

19. Billioti de Gage S, Moride Y, Ducruet T, et al. Benzodiazepine use and risk of Alzheimer's disease: case-control study. BMJ 2014;349:g5205.

20. American Geriatrics Society 2015 Updated Beers Criteria for potentially inappropriate medication use in older adults. $J$ Am Geriat Soc 2015;63:2227-46.

21. Finkle WD, Der JS, Greenland S, et al. Risk of fractures requiring hospitalization after an initial prescription for zolpidem, alprazolam, lorazepam, or diazepam in older adults. J Am Geriatr Soc 2011;59:1883-90.

22. Paterniti S, Dufouil C, Alpérovitch A. Long-term benzodiazepine use and cognitive decline in the elderly: the Epidemiology of vascular aging study. J Clin Psychopharmacol 2002;22:285-93.

23. Allain $\mathrm{H}$, Bentué-Ferrer $\mathrm{D}$, Polard $\mathrm{E}$, et al. Postural instability and consequent falls and hip fractures associated with use of hypnotics in the elderly: a comparative review. Drugs Aging 2005;22:749-65.

24. Cotroneo A, Gareri P, Nicoletti N, et al. Effectiveness and safety of hypnotic drugs in the treatment of insomnia in over 70-year old people. Arch Gerontol Geriatr 2007;44:121-4.

25. Tannenbaum C. Effect of age, education and health status on community dwelling older men's health concerns. Aging Male 2012;15:103-8.

26. Tannenbaum C, Mayo N, Ducharme F. Older women's health priorities and perceptions of care delivery: results of the WOW health survey. CMAJ 2005;173:153-9.
27. Anderson K, Stowasser D, Freeman C, et al. Prescriber barriers and enablers to minimising potentially inappropriate medications in adults: a systematic review and thematic synthesis. BMJ Open 2014;4:e006544.

28. Pawson $\mathrm{R}$. The science of evaluation: a realist manifestoAlA. Sage 2013.

29. Martin P, Tamblyn R, Ahmed S, et al. An educational intervention to reduce the use of potentially inappropriate medications among older adults (EMPOWER study): protocol for a cluster randomized trial. Trials 2013;14:80.

30. Tannenbaum C, Martin P. Sedative-Hypnotic medication DePrescribing brochure. Montréal ludgd,2014. http://www.criugm.qc. $\mathrm{ca} /$ fichier/pdf/BENZOeng.pdf

31. Hein G. Institute for inquiry constructivist learning theory. Institute for Inquiry available . $1991 \mathrm{http}: / /$ wwwexploratoriumedu/ifi/resources/ constructivistlearninghtmlS

32. Festinger L. A Theory of Social comparison processes. Human Relations 1954;7:117-40.

33. Michie S, van Stralen MM, West R. The behaviour change wheel: a new method for characterising and designing behaviour change interventions. Implement Sci 2011;6:42.

34. JW C, P-C V. Designing and conducting mixed methods research. Thousand Oaks, CA: Sage Publications, 2007.

35. Horne R, Weinman J, Hankins M. The beliefs about medicines questionnaire: the development and evaluation of a new method for assessing the cognitive representation of medication. Psychol Health 1999;14:1-24.

36. Bélanger L, Morin CM, Bastien C, et al. Self-efficacy and compliance with benzodiazepine taper in older adults with chronic insomnia. Health Psychol 2005;24:281-7.

37. McDowell I. Measuring health: a guide to rating scales and questionnaires. 3rd ed. New York, NY: Oxford University Press; US, 2006.

38. Sandelowski M. Combining qualitative and quantitative sampling, data collection, and analysis techniques in mixed-method studies. Res Nurs Health 2000;23:246-55.

39. Ellis P. The Essential Guide to effect sizes: statistical Power, MetaAnalysis, and the interpretation of Research results. Cambridge: Cambridge University Press, 2010.

40. Braun V, Clarke V. Using thematic analysis in psychology. Qual Res Psychol 2006;3:77-101.

41. O'Cathain A, Murphy E, Nicholl J. Three techniques for integrating data in mixed methods studies. BMJ 2010;341:c4587.

42. Farmer T, Robinson K, Elliott SJ, et al. Developing and implementing a triangulation protocol for qualitative health research. Qual Health Res 2006:16:377-94.

43. Prochaska J. Transtheoretical Model of Behavior Change. In: Gellman M, Turner JR, eds. Encyclopedia of behavioral medicine New York NY: Springer, 2013.

44. West R. Time for a change: putting the transtheoretical (Stages of Change) Model to rest. Addiction 2005;100:1036-9.

45. Cassel CK, Guest JA. Choosing wisely: helping physicians and patients make smart decisions about their care. JAMA 2012;307:1801-2.

46. Sunny C, McDonald DW, Landay JA. Theory-driven design strategies for technologies that support behavior change in everyday life. New York NY: ACM.

47. Johnson B, Christensen LB. Educational research: quantitative, qualitative, and mixed approaches. 5th ed, 2014.

48. Hilmer SN, Gnjidic D, Le Couteur DG. Thinking through the medication list - appropriate prescribing and deprescribing in robust and frail older patients. Aust Fam Physician 2012;41:924.

49. Holmes HM, Todd A. Evidence-based deprescribing of statins in patients with advanced illness. JAMA Intern Med 2015;175:701-2.

50. Bem DJ. Self-perception: an alternative interpretation of cognitive dissonance phenomena. Psychol Rev 1967;74:183-200.'

51. Dalkin SM, Greenhalgh J, Jones D, et al. What's in a mechanism? development of a key concept in realist evaluation. Implement Sci 2015;10:49.

52. Pawson R, Tilley N. Realistic evaluation. London: SAGE, 1997. 